\title{
INFLUÊNCIA DO SOMBREAMENTO NO DESENVOLVIMENTO DE MUDAS DE LOURO PIRARUCU (Licaria canella (Meissn.) Kosterm.)
}

\author{
Antonio M. PINTO' ${ }^{1}$, Vania P. VARELA ${ }^{1}$, Lúcio F. P. BATALHA ${ }^{1}$
}

RESUMO - Com o objetivo de avaliar o efeito dos níveis de sombreamento no desenvolvimento de mudas de Louro pirarucu (Licaria canella (Meissn.) Kosterm. - Lauraceae), conduziu-se um ensaio em viveiro na Reserva Florestal Adolfo Ducke. As sementes foram colocadas para germinar em caixas de madeira contendo areia lavada como substrato e posteriormente foi feito o transplante das mudas para sacos plásticos de cor preta. Utilizou-se quatro diferentes níveis de sombreamento. Os níveis de $30 \%, 50 \%$ e $70 \%$ de sombreamento foram obtidos por meio de telas de poliolefinas de cor preta e o nível de $0 \%$ a céu aberto. Foi empregado o delineamento em blocos ao acaso com parcelas subdivididas; os níveis de sombreamento constituiram as parcelas e os períodos de avaliação das mudas as subparcelas. Após 30,60 e 90 dias de permanência no viveiro, as mudas foram avaliadas quanto à altura, área foliar, razão de área foliar, peso de matéria seca da parte aérea e do sistema radicular. Baseando-se nos resultados obtidos, pode-se concluir: a) as mudas produzidas sob $50 \%$ de sombreamento apresentaram maiores valores de peso da parte aérea e do sistema radicular quando comparadas com $0 \%$ de sombreamento ; b) a altura, área foliar e razão de área foliar não foram influenciadas significativamente pelos níveis de sombreamento; c) as mudas com maior desenvolvimento em altura e maior peso do sistema radicular foram obtidas com 90 dias.

Palavras-chave: Sombreamento, Licaria canella, Louro Pirarucu

Influence of the Shading on Growth Seedlings of Louro Pirarucu (Licaria canella (Meissn.) Kosterm.).

ABSTRACT - This paper evaluates the shading effect on seedlings of "Louro Pirarucu" (Licaria canella (Meissn) Kosterm. - Lauraceae). The trial was set up in the nursery at Adolph Ducke forest reserve. The seeds were germinated in wooden boxes with washed sand as a substrate. After seed germination, the seedlings were transplanted to black polyethylene bags. Four levels of shading $(0 \%$, $30 \%, 50 \%$ and $70 \%$ ) is analyzed. Shading treatments were obtained with plastic shade clothes of different meshes. The experimental design was the randomized blocks with split-plot. Shading levels were considered plots and period of harvesting as sub-plots. Evaluations of potted seedlings were done after 30,60 and 90 days for the following parameters: height, leaf area, leaf area ratio, aerial dry weight and root dry weight. The results suggest that: a) potted seedlings grown under $50 \%$ of shade presented higher values of aerial weight and root weight than plants under full sunlight radiation; b) height, leaf area and leaf area ratio were not significantly influenced by shading treatments; c) higher values of height and root weight were observed after 90 days.

Key words: Shading, Licaria canella, Louro Pirarucu

\section{INTRODUÇÃO}

A exploração desordenada e seletiva das espécies florestais que vem ocorrendo ultimamente na
Amazônia em decorrência da demanda para atender o mercado nacional e internacional tem resultado em perdas de grandes populações antes mesmo que sejam conhecidas suas

1 Instituto Nacional de Pesquisas da Amazônia, Caixa Postal 478, 69011-970 - Manaus, Amazonas, Brasil. 
silviculturais. Os mecanismos de regeneração das espécies florestais da região têm sido pouco estudados e existe carência de informações que permitam um melhor entendimento dos sistemas de regeneração destas espécies, principalmente no que se refere aos mecanismos reprodutores.

Estudos ecofisiológicos que indiquem a influência dos fatores ambientais na distribuição, sobrevivência e desenvolvimento das espécies florestais e que auxiliem na compreensão de seu papel no ecossistema tornam-se, portanto, fundamentais. O plantio de espécies florestais, seja com a finalidade econômica ou conservacionista, requer uma série de cuidados que dependem do conhecimento prévio de suas características fisiológicas e exigências ecológicas nas diversas etapas de seu ciclo vital.

O louro pirarucu (Licaria canella), segundo SILVA et al. (1977), é uma árvore mediana a grande $(15-40 \mathrm{~m}$ de altura), encontrada na mata de terra firme e em solo argiloso. Embora sejam poucas as informações sobre as aplicações da espécie em estudo, procurou-se levar em consideração a utilização do gênero Licaria (Lauraceae) que é formecedor de uma das melhores madeira entre os louros usadas em construções em geral.

O desconhecimento sobre métodos de produção de mudas de louro pirarucu (Licaria canella) com melhor padrão de qualidade constitui um dos fatores limitantes ao amplo aproveitamento desta espécie nos programas silviculturais.
É de suma importância realizar estudos relativos ao crescimento e qualidade de mudas em viveiros, no sentido de fornecer informações básicas sobre o desenvolvimento de espécies em condições de plantios. Alguns trabalhos têm apresentado resultados sobre a qualidade de mudas de regiões temperadas. Entretanto, pela revisão de literatura realizada, há carência de informações para as espécies florestais de regiões tropicais e como consequiência, há um campo vasto de estudos a serem realizados.

GORDON (1969), em estudos com Betula alleghaniensis, verificou que o maior desenvolvimento em altura ocorreu nas mudas sombreadas. Observou maior área foliar total por planta quando sob sombreamento mais elevado.

FAIRBAIRN \& NEUSTEIN (1970), encontraram melhor desenvolvimento em altura para as mudas de Pseudotsuga menziesii, Tsuga heterophylla, Abies grandis e Picea abies produzidas com pouco sombreamento; entretanto, para as de Picea sitchensis, observaram maior altura quando não foram sombreadas.

FERREIRA (1977), estudando o efeito do sombreamento na produção de mudas de quatro espécies florestais nativas, observou para Schizolobium parahyba e Hymeneae stigonocarpa, maior diâmetro ao colo quando as mudas foram produzidas sem sombreamento, ao passo que para Pelthophorum dubium e Enterolobium contortisiliguum não foi verificada diferença significativa. 
Segundo INOUE (1977), para um rápido crescimento no estágio juvenil, a espécie Cedrella spp. necessita de um sombreamento. Esse autor afirma que o crescimento depende não só da intensidade luminosa, mas também da duração da radiação de energia (hora de insolação diária).

STURION (1980), desenvolvendo estudos com Prunus brasiliensis, verificou que entre os níveis de sombreamento testados $(0 \%, 30 \%$ e $60 \%)$, as maiores sobrevivências e altura foram obtidas com as mudas conduzidas sob $30 \%$ e $60 \%$ de sombreamento. Observou também, maior diâmetro do colo, maior peso da matéria seca do sistema radicular e da parte aérea nas mudas conduzidas a ceu aberto.

AMO (1985) observou que as diferenças de luz quanto a sua intensidade tem, nas condições naturais, efeito mais significativo no crescimento das plantas do que a sua qualidade, principalmente quanto ao acúmulo de matéria seca.

VARELA \& SANTOS (1992), trabalhando com mudas de angelim pedra (Dinizia excelsa), observaram maior desenvolvimento em altura quando avaliadas com 90 dias e quando cultivadas sob $30 \%$ e $50 \%$ de sombreamento. Observaram maiores valores de peso de matéria seca da parte aérea e do sistema radicular nestes níveis.

Betula alleghaniensis e Schizolobium parahyba apresentaram maior área foliar total por planta quando sob sombreamento mais elevado (GORDON, 1969; FERREIRA, 1977).

Estudando o efeito do sombreamento sobre o crescimento das mudas de três espécies florestais, POGGIANI $\boldsymbol{e t}$ al. (1992), observaram nas plântulas de Piptadenia rigida, incremento significativo do peso das folhas secas e da área foliar quando exposta a $80 \%$ de sombra.

O presente estudo tem como. objetivo obter informações sobre o crescimento de mudas de louro pirarucu (Licaria canella) cultivadas sob diferentes níveis de sombreamento e estabelecer condições mais adequadas para o seu desenvolvimento.

\section{MATERIAL E MÉTODOS}

O estudo foi desenvolvido no período de junho de 1992 a janeiro de 1993 no viveiro de produção de mudas da Reserva Florestal Adolfo Ducke, localizada no $\mathrm{km} 26$ da rodovia AM010, em Manaus-AM. A reserva está compreendida entre as coordenadas geográficas de $03^{\circ} 00^{\prime} 02^{\prime \prime}$ e $03^{\circ} 08^{\prime} 00^{\prime \prime}$ de latitude sul e $59^{\circ} 52^{\prime} 40^{\prime \prime}$ e $59^{\circ} 58^{\prime} 00^{\prime \prime}$ de longitude oeste.

O clima local, pela classificação de Köppen, é do tipo Afi. RIBEIRO (1976), com bases em dados meteorológicos de nove anos da Reserva Ducke, constatou que a temperatura média para o mes mais frio nunca é inferior a $18^{\circ} \mathrm{C}$, a precipitação média anual é de $2000 \mathrm{~mm}$ e ocorrem duas estações distintas: a chuvosa estendendo-se de novembro a maio e a seca de junho a outubro.

Trabalhos preliminares: determinação da germinação e teste adicionais.

As sementes de louro pirarucu foram procedentes da Reserva Florestal 
Adolfo Ducke e coletadas, de uma única matriz, em 16 de junho de 1992. Após a homogeneização do lote, foram retiradas amostras e enviadas ao laboratório de sementes do Instituto Nacional de Pesquisas da Amazônia para que fossem determinada a germinação e efetuados os testes adicionais. As sementes apresentaram as seguintes características por ocasião do experimento:

. Peso de mil sementes $=15.687 \mathrm{~g}$

. Teor de umidade $=33,25 \%$

. Porcentagem de germinação $=82 \%$

\section{Condições de cultivo das mudas}

As sementes foram coletadas no mesmo local da experimentação em 16 de junho de 1992. A semeadura foi realizada no dia 24 de junho de 1992 em caixas de madeira de $100 \times 47 \times$ $21 \mathrm{~cm}$ contendo areia lavada como substrato, sob galpão coberto com telhas transparentes. Na repicagem, realizada em 13 de outubro de 1992 , foram utilizados sacos plásticos de cor preta com $16,5 \mathrm{~cm}$ de diâmetro por $28,5 \mathrm{~cm}$ de altura e uma mistura de areia e barro na proporção de 2:1. Dez dias após, procedeu-se o transporte das mudas para os canteiros.

No viveiro foram utilizados quatro níveis de sombreamento: $\mathrm{S}_{1}=$ $30 \%, \mathrm{~S}_{2}=50 \%$ e $\mathrm{S}_{3}=70 \%$ obtidos com tela de poliolefina de cor preta e $\mathrm{S}_{4}=0 \%$, a céu aberto. As telas recobriam porções superiores e laterais de armações de madeira de 5,0 x 1,0 x $0,5 \mathrm{~m}$. No decorrer do experimento, os canteiros foram irrigados diariamente e as ervas daninhas eliminadas.
As mudas foram distribuídas no viveiro, nas condições acima mencionadas, em blocos ao acaso, com parcelas subdivididas, utilizando-se duas repetições de 30 mudas por tratamento. As parcelas foram constituídas pelo tipos de sombreamento e as sub parcelas pelos períodos de observações ( 30 , 60 e 90) dias.

\section{Avaliação da qualidade das mudas}

A avaliação do crescimento foi feita mensalmente através da determinação de várias características relativas ao desenvolvimento vegetativo das mudas. Foram avaliados os seguintes parâmetros:

. Altura total da muda (cm) - avaliada até a inserção da última folha com o auxílio de uma régua milimetrada.

Área foliar $\left(\mathrm{dm}^{2}\right)$ - avaliada através de um medidor de área foliar portátil LI-COR Mod. LI-3000A.

. Peso (g) da parte aérea, sistema radicular e total, após secagem em estufa. As mudas seccionadas em duas partes (aérea e radicular) foram colocadas para secar em estufa a $75^{\circ} \mathrm{C}$ por 48 horas e posteriormente pesadas em balança analítica com precisão de $0,001 \mathrm{~g}$ para determinação do peso.

- Razão da área foliar $\left(\mathrm{dm}^{2} / \mathrm{g}\right)$ avaliada através da relação entre área foliar e peso total.

As determinações acima foram feitas tomando-se, ao acaso, amostras de cinco mudas de cada condição, por repetição a intervalos de 30,60 e 90 dias após a transferência das mudas para o viveiro. 


\section{RESULTADOS E DISCUSSÃO}

Os resultados médios de altura, área foliar, pesos da parte aérea, do sistema radicular e, razão de área foliar obtidos em função dos períodos de avaliação, são apresentados na Tabela 1 . Observou-se que o peso da parte aérea não foi influenciado significativamente pelos períodos nos quais as mudas permaneceram no viveiro. Com relação à altura, verificou-se que o maior desenvolvimento foi observado nas mudas obtidas com 90 dias e nas mudas obtidas com 30 e 60 dias não foram constatadas diferenças significativas. VARELA \& SANTOS (1992), analisando a influência do sombreamento na produção de mudas de angelim (Dinizia excelsa), também observaram maior desenvolvimento em altura quando as mudas foram obtidas com 90 dias.

Os pesos do sistema radicular obtidos com 30 e 60 dias não mostraram diferenças significativas entre si, entretanto foram inferiores aos obtidos com 90 dias de observação.

Analisando-se a razão de área fo- liar na Tabela 1, verificou-se que embora não exista diferença significativa entre 30 e 60 dias, foram obtidos valores estatisticamente superiores quando comparados com os obtidos com 90 dias.

As médias dos dados de altura, área foliar, pesos da parte aérea, do sistema radicular e razão de área foliar em função de diferentes níveis de sombreamento, encontram-se na Tabela 2 . Verificou-se que a altura das mudas não foi influenciada significativamente pelo sombreamento e pela interação sombreamento-período. FERREIRA et al. (1981), no entanto, observaram variações acentuadas na altura de Pinus insulares em função dos níveis de sombreamento; as mudas que atingiram altura mais elevadas foram as produzidas sob $70 \%$ de sombreamento. Nos estudos em viveiro com mudas de Pinus insulares, com dois anos de idade, FAIRBAIRN \& NEUSTEIN (1970) encontraram melhores respostas em altura quando nao foram sombreadas.

Analisando-se a influência do sombreamento sobre o peso da parte aérea e do sistema radicular, conforme

Tabela 1. Média de altura, área foliar, matéria seca da parte aérea, sistema radicular e razão de área foliar, obtidas após 30, 60 e 90 dias de permanência no viveiro.

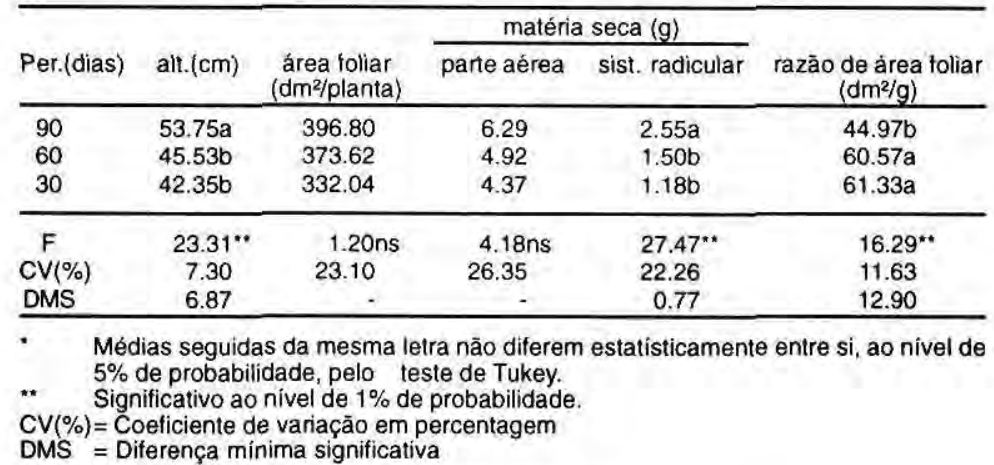


Tabela 2. Média de altura, área foliar, matéria seca da parte aérea, sistema radicular e razão de área foliar, obtidas em função do sombreamento.

\begin{tabular}{|c|c|c|c|c|c|}
\hline \multirow[b]{2}{*}{ Somb. } & \multirow[b]{2}{*}{ alt. (cm) } & \multirow[b]{2}{*}{$\begin{array}{c}\text { área foliar } \\
\text { (dm²/planta) }\end{array}$} & \multicolumn{2}{|c|}{ matéria seca $(\mathrm{g})$} & \multirow{2}{*}{$\begin{array}{c}\text { razāo de área foliar } \\
\left(\mathrm{dm}^{2} / \mathrm{g}\right)\end{array}$} \\
\hline & & & parte aérea & sist. radicular & \\
\hline $\begin{array}{l}0 \% \\
30 \% \\
50 \% \\
70 \%\end{array}$ & $\begin{array}{l}45.03 \\
44.63 \\
42.98 \\
49.70\end{array}$ & $\begin{array}{l}323.96 \\
354.26 \\
425.92 \\
365.80\end{array}$ & $\begin{array}{l}4.08 \mathrm{~b} \\
5.31 \mathrm{ab} \\
6.47 \mathrm{a} \\
4.92 \mathrm{ab}\end{array}$ & $\begin{array}{l}1.33 \mathrm{~b} \\
1.73 \mathrm{ab} \\
2.09 \mathrm{a} \\
1.81 \mathrm{ab}\end{array}$ & $\begin{array}{l}61.59 \\
51.70 \\
51.03 \\
58.17\end{array}$ \\
\hline $\begin{array}{l}\text { Fs } \\
\text { Fsp } \\
\text { CV }(\%) \\
\text { DMS } \\
\end{array}$ & $\begin{array}{l}5.88 \mathrm{~ns} \\
0.90 \mathrm{~ns} \\
5.88 \\
\end{array}$ & $\begin{array}{c}5.72 \mathrm{~ns} \\
0.28 \mathrm{~ns} \\
11.91 \\
\end{array}$ & $\begin{array}{l}31.32^{\star *} \\
0.54 \mathrm{~ns} \\
8.40 \\
2.17 \\
\end{array}$ & $\begin{array}{c}14.50^{*} \\
1.60 \text { ns } \\
11.49 \\
0.56 \\
\end{array}$ & $\begin{array}{r}3.55 \mathrm{~ns} \\
0.90 \mathrm{~ns} \\
11.96 \\
\end{array}$ \\
\hline $\begin{array}{l}* \\
\text { Fs, Fsp } \\
\text { CV } \\
\text { DMS }\end{array}$ & $\begin{array}{l}\text { Médias seguid } \\
\text { de } 5 \% \text { de prob } \\
\text { Significativo ao } \\
=\text { Valores de } F \\
\text { (sombreament } \\
=\text { Coeficiente } d \\
=\text { Difirença mir }\end{array}$ & $\begin{array}{l}\text { las da mesma } \\
\text { labilidade, pelo } \\
\text { nivel de } 1 \% \text { d } \\
\text { referentes aos } \\
\text { to-periodo) } \\
\text { de variação em } \\
\text { nima significati }\end{array}$ & $\begin{array}{l}\text { etra não difere } \\
\text { teste de Tuk } \\
\text { e probabilidad } \\
\text { niveis de som } \\
\text { percentagem }\end{array}$ & amento e inte & $\begin{array}{l}\text { entre si, ao nivel } \\
\text { ção }\end{array}$ \\
\hline
\end{tabular}

mostra a tabela 2 , verificou-se que os tratamentos de 30,50 e $70 \%$ de sombreamento não mostraram diferenças significativas entre si em relação a estes parâmetros; entretanto, observou-se que o tratamento sob $50 \%$ de sombreamento proporcionou maiores valores quando comparados com 0\%. Para mudas de andiroba (Carapa guianensis), VARELA \& MARTINS (no prelo), entretanto, verificaram um decréscimo no peso da matéria seca da parte aérea com o aumento do sombreamento. STURION \& IEDE (1982), trabalhando com mudas de Ocotea porosa, encontraram maior produção de matéria seca do sistema radicular quando produzidas a céu aberto e sob $30 \%$ de sombreamento. Verificaram que não houve diferenças significativas entre os pesos de matéria seca da parte aérea das mudas em função dos níveis de sombreamento. FERREIRA et al. (1981), observaram valores maiores na produção de matéria seca da raiz quando as mudas de Pinus insulares não foram submetidas ao sombreamento.
Com relação à área foliar, conforme mostra a Tabela 2, não foram constatadas diferenças significativas quanto aos níveis de sombreamento, e a interação níveis de sombreamento-período. Em mudas de Theobroma cacao, com 18 meses de idade, foi observado aumento relativo da área foliar por planta com o sombreamento (MURRAY \& NICHOLS, 1966). O mesmo foi observado para Acer saccharum e Betula alleghaniensis (GORDON, 1969; LOGAN \& KROTKOV, 1969). Observou-se que não houve diferença significativa entre a razão de área foliar das mudas em função dos níveis de sombreamento e da interação sombreamentoperíodo. FERREIRA (1977), no entanto, observou que a área foliar e razão de área foliar, para o Guapuruvu Schizolobium parahyba, foram maiores sob $70 \%$ de sombreamento do que $0 \%$.

\section{CONCLUSÕES}

- Mudas com 90 dias de 
permanência no viveiro apresentaram maior desenvolvimento em altura e maior peso do sistema radicular em comparação com as de 30 e 60 dias.

- As mudas com 30 e 60 dias apresentaram maior razão de área foliar que as de 90 .

- Para área foliar e peso da parte aérea não ocorreram diferenças significativas em função dos períodos

- A altura, área foliar e razão de área foliar não foram influenciadas significativamente pelos níveis de sombreamento e pela interação sombreamento-período.

- As mudas produzidas sob $50 \%$ de sombreamento apresentaram maiores valores de peso da parte aérea e do sistema radicular quando comparadas com as produzidas sob $0 \%$ de sombreamento.

\section{AGRADECIMENTOS}

Agradecemos ao funcionário PEDRO CÁSSIO MENDES DE ALBUQUERQUE pelo auxílio na coleta dos dados.

\section{Literatura citada}

AMO, S. R. Del. 1985. Alguns aspectos de la influência de la luz sobre el crescimento de estados juveniles de espécies primárias. In: GOMES-POMPA, A.L. Reneracion de selvas 2xico, Editora Alhambra Mexicana S.A. p. 79-9

FAIRBAIRN, W.A.; NEUSTEIN, S.A. 1970. Study of response of certain coniferous species to light intensity. Forestry, 43(1):57-71.
FERREIRA, M.G.M. 1977. Efeito do sombreamento na produção de mudas de quatro espécies florestais nativas. Viçosa, Universidade Federal de Viçosa, (Tese M.Sc.). 42p.

FERREIRA, M.G.M.; CÂNDIDO J.F; SILVA, D.A.; COLODETTE, J.L. 1981. Efeito do sombreamento e da densidade de semente sobre o desenvolvimento de mudas de Pinus insulares Endlicher e seu crescimento inicial no campo. Revista Floresta, 2(1):53-61.

GORDON, J.C. 1969. Effect of shade on photosynthesis and dry weight distribution on yellow birch. (Betula alleghaniensi Britton) seedlings. Ecology, 50(5):924-6.

INOUE, M.T. 1977. A auto-ecologia do gênero Cedrela: efeitos na fisiologia do crescimento no estágio juvenil em função da intensidade luminosa. Floresta, Curitiba, 6(2):58-61.

LOGAN, K.T.; KROTKOV, G. 1969. Adaptations of the photossynthetic mechanism of sugar maple (Acer saccharum) seedlings grown in various light intensities. Physiologia Plantarum, 22(1):104-16.

MURRAY, D.B.; NICHOLS, R. 1966. Light, shade and growth in some tropical plants. In: BAINBRIDGE, R.; EVANS, G.C. \& RACKHAM, O. (Eds.). Light as an ecological factors. Blackwell, $\mathrm{Ox}$ ford, p 249-3.

POGGIANI, F.; BRUNI, S.; BARBOSA, E.S.Q. 1992. Efeito do sombreamento sobre o crescimento das mudas de três espécies florestais. Anais do $2^{\circ}$ Congresso Nacional sobre Essências Nativas. São Paulo, 564-569.

RIBEIRO, M.N.G. 1976. Aspectos climatológicos de Manaus. Acta Amazonica, 6(2):229-33, 1976.

SILVA, M. F. ; LISBOA, P. L. B.; LISBOA, R. C. L. 1977. Nomes vulgares de plantas amazônicas. Belém-PA, INPA, 222 p.

STURION, J.A. 1980. Influência da profundidade de semeadura, cobertura do canteiro e sombreamento, na formação 
de mudas de Prunus brasiliensis Schott ex Spreng. Boletim de Pesquisa Florestal (1): 50-68.

STURION, J.A.; IEDE, E.T. 1982. Influência da profundidade de semeadura, cobertura do canteiro e sombreamento na formação de mudas de Ocotea porosa (NEES). Silvicultura, 28:513-6.
VARELA, V.P.; SANTOS, J. 1992. Influência do sombreamento na produção de mudas angelim pedra (Dinizia excelsa Ducke). Acta Amazonica, 22(3):407-411

VARELA, V.P.; MARTINS, R.O. Influência da profundidade de semeadura e do tipo de sombreamento na produção de Andiroba (Carapa guianensis Aubl.) Pesq. Agrop. Bras. (no prelo). 